\title{
Quiste nasolabial: reporte de un caso y revisión de la literatura
}

\section{Nasolabial cyst: a case report and review of the literature}

\author{
Chrcanovic BR*, López Alvarenga R**, Souza LN***, De Paula AMB****, \\ Freire-Maia B*****
}

\section{RESUMEN}

El quiste nasolabial es un raro quiste no odontogénico, su origen embriológico es poco claro. Son lesiones de crecimiento extraóseo, que se presentan como una tumefacción que eleva el ala nasal, indoloro, desplazable y fluctuante. Se localiza en la apófisis alveolar cercana a la base del ala de la nariz. El tratamiento consiste en la enucleación quirúrgica de la lesión. En este artículo se presenta un caso de quiste nasolabial, que acomete a una paciente de sexo femenino de 51 años de edad, de la cual se discuten los datos clínicos, aspectos diagnósticos, imagenológicos, histopatológicos, así como su tratamiento.

Palabras clave: Quiste nasolabial, quiste nasoalveolar, quiste no odontogénico.

\section{SUMMARY}

The nasolabial cyst is a rare nonodontogenic cyst with its embryological origin little clear. They are extraosseous lesions with growth that appears like a swelling that lifts the nasal wing, painless, sliding and fluctuating. It is located in the alveolar apophysis near the base of the wing of the nose. The treatment consists of surgical enucleating of the lesion. This article presents a case of nasolabial cyst in a female patient of 46 years age, describing clinical, diagnostic, imaging, and histopathological aspects as well as the treatment employed.

Key words: Nasolabial cyst, nasoalveolar cyst, nonodontogenic cyst.

Fecha de recepción: 2 de noviembre de 2009.

Aceptado para publicación: 20 de noviembre de 2009.

* Departamento del Servicio de Cirugía y Traumatología Oral y Maxilofacial del Hospital María Amélia Lins (HMAL) de Belo Horizonte, Brasil.

** Licenciado en Odontología por la universidad del Centro Universitario Newton Paiva, Belo Horizonte, Brasil.

*** Prof. Adjunto de Cirugía Oral y Maxilofacial, Centro Universitario Newton Paiva, Belo Horizonte, Brasil.

**** Prof. Adjunto de Patología Oral de la Universidad Estadual de Montes Claros, Montes Claros, Belo Horizonte, Brasil.

***** Prof. Asistente de Cirugía Oral y Maxilofacial, Facultad de Odontología de la Pontificia Universidad Católica, Belo Horizonte, Brasil.

Chrcanovic BR, López Alvarenga R, Souza LN, De Paula AMB, Freire-Maia B. Quiste nasolabial: reporte de un caso y revisión de la literatura. Av. Odontoestomatol 2011; 27 (3): 123-127.

\section{INTRODUCCIÓN}

La primera descripción de los quistes nasolabiales, también denominados quistes nasoalveolares y quistes de Klestadt, se debe a Zuckerkandl en 1882, sien- do publicado el primer caso en la literatura en 1892 por Chatelier $(1,2)$.

El quiste nasolabial es un raro quiste no odontogénico de tejidos blandos (extraóseo). Se localiza en la 
parte anterior del maxilar superior, debajo del ala de la nariz y en la profundidad del surco nasolabial (3). Se manifiesta con más frecuencia en mujeres $(3,5: 1)$, entre la $3^{\mathrm{a}}$ y $6^{\mathrm{a}}$ década de la vida (4).

Clínicamente, el quiste nasolabial se presenta como una tumefacción de crecimiento lento, asintomática, de consistencia blanda y elevación del ala de la nariz. Suelen ser unilaterales, aunque se han publicado casos bilaterales $(4,5,6)$. En los exámenes radiográficos no suelen presentar expresión, solo da positivo en algunos casos concretos $(4,5,6)$.

Histológicamente podemos observar una cavidad quística constituida generalmente por un epitelio y rodeado por una pared conectiva. El tipo más frecuente del epitelio es el columnar pseudoestratificado que muestra un número de células mucosas (caliciformes) y células ciliadas $(5,7,8)$.

El tratamiento del quiste nasolabial se hace a través de la enucleación quirúrgica completa de la lesión $(5,7,8)$.

El objetivo de este trabajo es presentar un caso de quiste nasolabial, en el cual se discuten los aspectos característicos del caso y hacer una breve revisión de la literatura.

\section{CASO CLÍNICO}

Paciente femenino de 51 años de edad, sin antecedentes patológicos de interés, fue remitido al Servicio de Cirugía y Traumatología Buco maxilofacial del Hospital María Amélia Lins (HMAL) por su odontólogo, por presentar asimetría nasal y obstrucción nasal con aproximadamente 1 año de evolución.

En el examen extraoral se evidenció prominencia del ala nasal derecha. Durante la exploración intraoral se observó un abombamiento indoloro del surco gingivolabial de consistencia blanda y con una mucosa de características normales al tejido adyacente (Fig. 1). La paciente no refiere ninguna sintomatología de rinorrea o epistaxis.

El examen radiográfico (Oclusal) reveló una imagen de reabsorción ósea en forma de taza en la pared

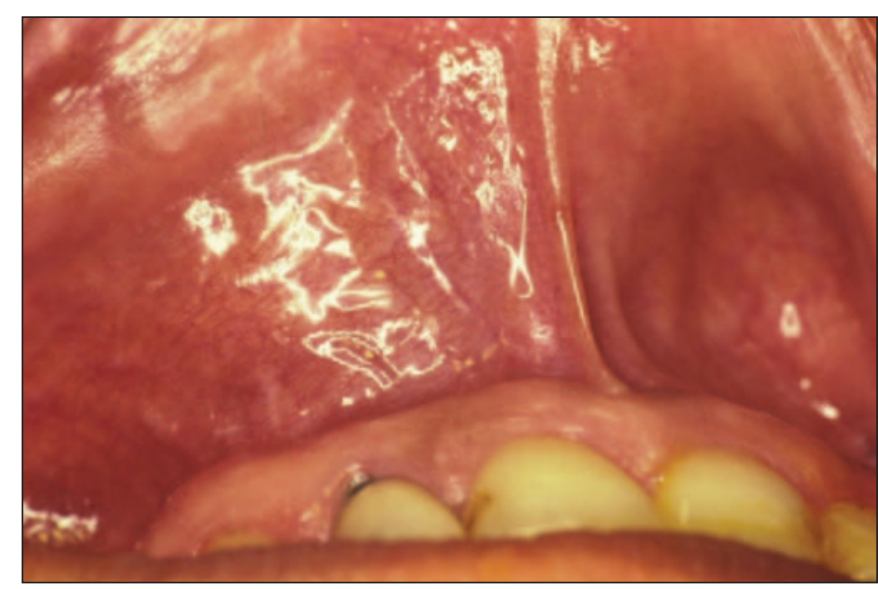

Fig.1. Imagen intraoral donde se observa aumento de volumen del surco gingivolabial del lado derecho.

nasal lateral (Fig. 2). En la tomografía computarizada, utilizando una ventana para tejido blando, se observó claramente alteración de la arquitectura ósea de la pared anterior del maxilar superior, en el que se aprecia una lesión de tejidos blandos, de forma redondeada y circunscrita de 2-3 cm de diámetro de características quísticas (Fig. 3).

Una vez completado el examen clínico, el análisis de los exámenes imagenológicos y de vitalidad pulpar, se determina como diagnóstico clínico quiste nasolabial.

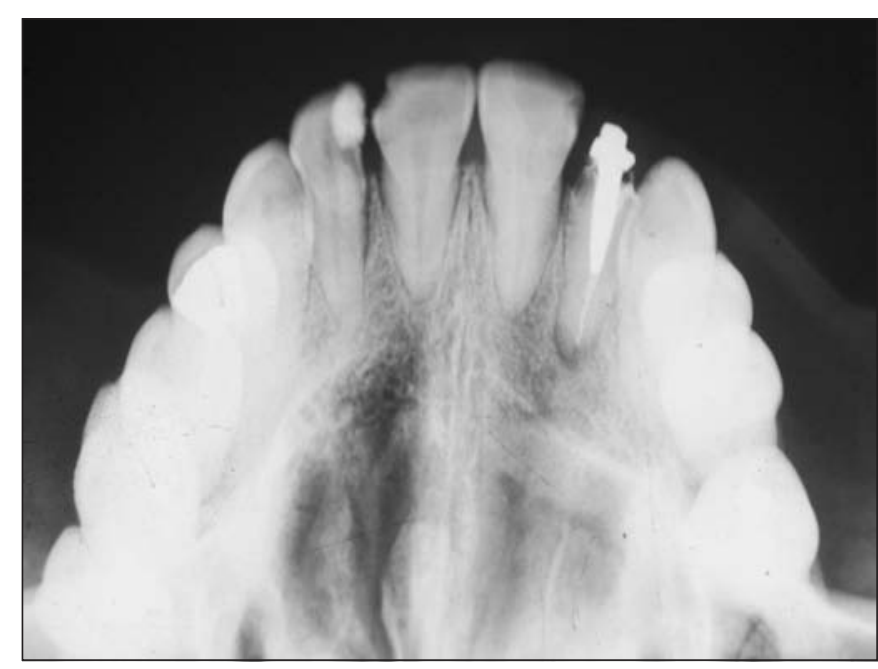

Fig. 2. Radiografía oclusal superior; muestra una erosión del hueso de la pared nasal lateral y una elevación del suelo de la fosa nasal. 


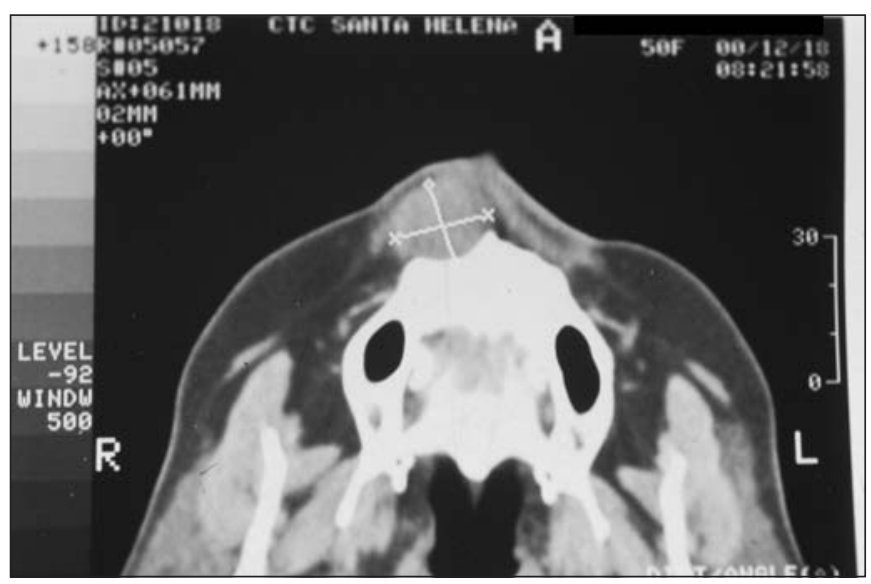

Fig. 3. Tomografía computarizada utilizando ventana para tejido blando en corte axial revelando alteración de la arquitectura ósea de la pared anterior del maxilar superior, en el que se aprecia una lesión de tejidos blandos, redondeada y circunscrita.

Bajo el diagnóstico clínico de quiste nasolabial, el paciente fue intervenido quirúrgicamente bajo anestesia general, en la cual, mediante un acceso intraoral sublabial se realizó una enucleación completa de la lesión (Fig. 4A). Se remitió el espécimen quirúrgico para el análisis anatomopatológico (Fig. 4B).

El informe anatomopatológico de la pieza operatoria, da a conocer una pared fibroconjuntiva revestida por tejido epitelial pseudoestratificado columnar con células caliciformes; confirmando el diagnóstico clínico de quiste nasolabial (Figs. 5 y 6).

El seguimiento del paciente con controles periódicos no mostró en 2 años signos de evidencia de recidiva.

\section{DISCUSIÓN}

El origen embriológico de los quistes nasolabiales son inciertos, aunque existen dos teorías principales. Una teoría considera que el quiste nasolabial es un quiste fisural que se origina en las líneas de fusión de los procesos lateral nasal, medial nasal y maxilares, supuestamente por retenciones de restos ectodérmicos. La segunda teoría considera que estos quistes se desarrollan debido al atrapamiento de tejidos ectodérmicos en el interior del conducto nasolacrimal desplazado $(7,8)$. En nuestro caso presentado,

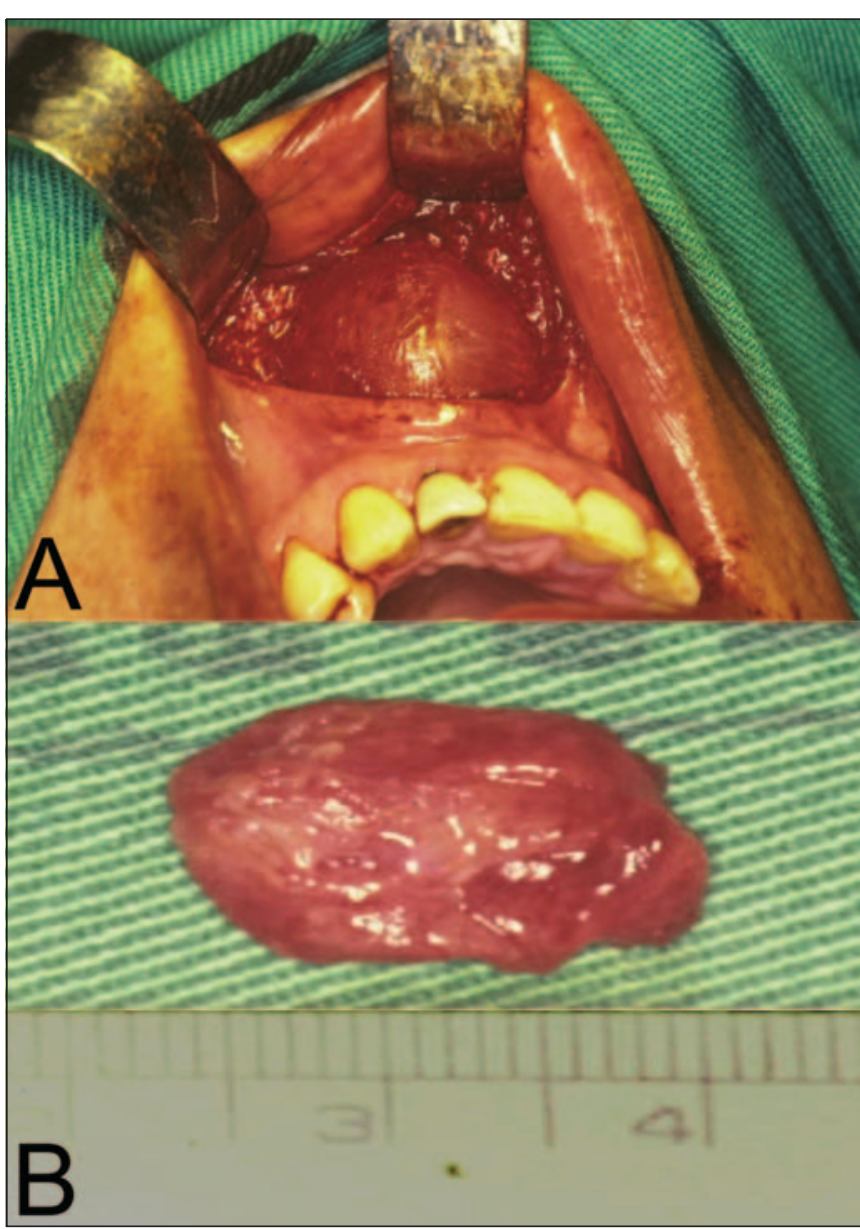

Fig. 4. A. Imagen intraoperatorio, durante el abordaje quirúrgico. B. Espécimen quirúrgico.

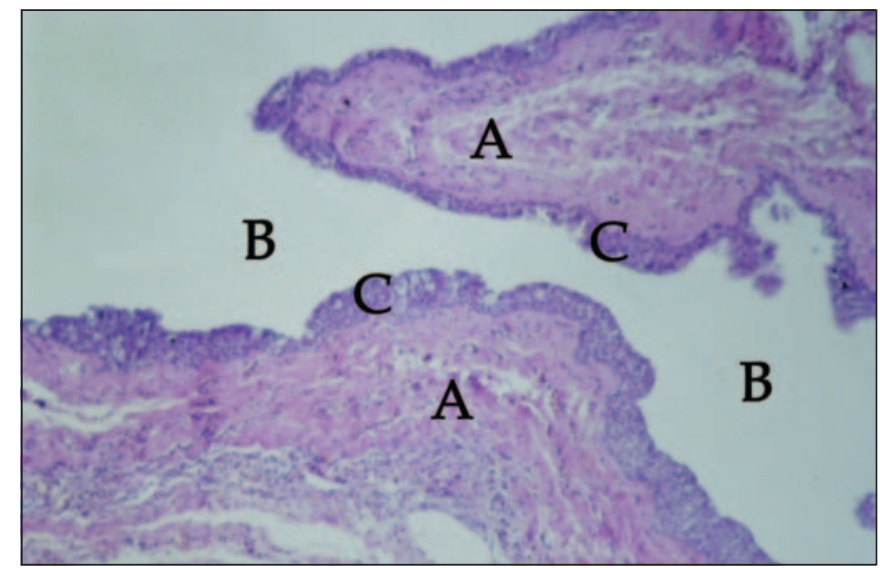

Fig. 5. Estudio anatomopatológico: cápsula fibrosa con un infiltrado inflamatorio crónico del tipo mononuclear (A) y una cavidad quística (B) revestido por un tejido epitelial (C). (HE 100x) 


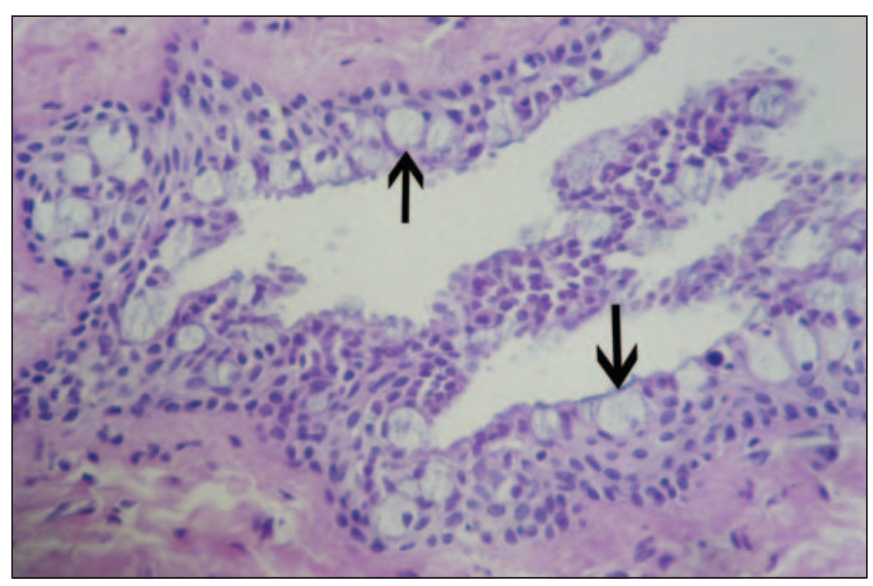

Fig. 6. Estudio anatomopatológico donde se observa un tejido epitelial pseudoestratificado columnar con células caliciformes. (HE 400x)

el informe anatomopatológico de la pieza operatoria, se observa una pared fibroconjuntiva revestida por tejido epitelial pseudoestratificado columnar con células caliciformes semejante al epitelio de revestimiento de la pared del conducto nasolacrimal.

Las manifestaciones clínicas consisten en una tumefacción de crecimiento lento, fluctuante, que eleva el cartílago alar, distorsiona la forma de la ventana nasal externa, oblitera el pliegue nasolabial y de dimensiones variables (2-3 cm) (9). Sin embargo, fue descrito un caso en la literatura de un quiste nasolabial gigante, en el cual se drenó $250 \mathrm{ml}$ de su contenido (10). La tumefacción suele ser asintomática, a menos que se infecte secundariamente, en cuyo caso puede haber un aumento repentino del tamaño, seguido por una descarga de pus a través de la nariz y provocar obstrucción nasal $(6,8)$. Algunos casos de gran expansión llegan a producir reabsorciones óseas en la apófisis alveolar por debajo de la abertura piriforme. La vitalidad de los dientes adyacentes está conservada $(6,8)$.

Los quistes nasolabiales son lesiones de los tejidos blandos situados adyacentes al proceso alveolar, por encima de los ápices de los incisivos. Debido a que se trata de una lesión de partes blandas, las radiografías convencionales pueden no mostrar cambio detectable alguno (11). Algunos casos la reabsorción del hueso subyacente (por presión) puede observarse radiográficamente (11). El uso de medios de contraste ha sido empleado para determinar la dimensión del quiste. Las pruebas necesarias deberían incluir bien tomografía computarizada o resonancia magnética $(6,8)$. Estos métodos complementarios son los que aportan las mejores imágenes de los tejidos blandos. En el caso presentado, los hallazgos imagenológicos fueron compatibles con los descritos en la literatura.

El quiste nasolabial infectado puede confundirse con un absceso dentoalveolar agudo. Para evitar este error, se debe comprobar la vitalidad de los dientes adyacentes. Además, se debe diferenciar este quiste de una forunculosis nasal, de quistes odontogénicos, quistes fisurales y granulomas periapicales $(6,8)$. Sin embargo, nunca hay que descartar el diagnóstico diferencial de un quiste nasolabial no infectado, los quistes de extravasación mucoso grande o un adenoma quístico salivar $(6,8)$.

Desde el punto de vista histológico, el quiste nasolabial está tapizado de epitelio pseudoestratificado que a veces es cilíndrico, frecuentemente con células caliciformes o de epitelio escamoso estratificado. Suele observarse una zona de tejido fibroso denso y homogéneo adyacente al revestimiento epitelial. En algunos casos se han descrito áreas de metaplasia escamosa, o un epitelio francamente escamoso y cambios apocrinos. También puede presentar inflamación si la lesión está infectada secundariamente (12).

El tratamiento habitual consiste en la resección quirúrgica completa por vía intraoral mediante una incisión sublabial $(3,7,13)$. Algunos autores proponen un abordaje endonasal con marsupialización, sin embargo, esta técnica tiene pocas ventajas (13). En general, los resultados de la enucleación de la lesión por la vía sublabial suelen ser excelentes. El protocolo utilizado para el tratamiento de este caso particular fue la enucleación completa de la lesión mediante un acceso intraoral sublabial.

El pronóstico del quiste nasolabial es excelente y las recurrencias son raras $(4,12)$.

El conocimiento de las características clínicas y imagenológicas del quiste nasolabial son necesarias para el correcto diagnostico y tratamiento adecuado. 


\section{CONCLUSIONES}

La revisión de la literatura y el caso clínico descrito permiten realizar las siguientes consideraciones:

- Los autores son bastantes uniformes con respecto a las características clínicas de los quistes nasolabiales, relatando la predilección de la lesión por el sexo femenino entre la $3^{\mathrm{a}}$ y $6^{\mathrm{a}}$ década de la vida, tumefacción de crecimiento lento, fluctuante y elevación del ala de la nariz.

- Tratándose de un quiste poco frecuente, se debe de analizar las patologías más frecuentes con las que se puede confundir y hay necesidad de hacer diagnóstico diferencial.

- Los exámenes complementarios deben incluir una tomografía computarizada con ventana para tejido blando o resonancia magnética. Estos métodos son los que aportan las mejores imágenes para una correcta evaluación y delimitación de la lesión, así como para establecimiento del tratamiento.

\section{BIBLIOGRAFÍA}

1. Wesley RK, Scannel T, Nathan LE. Nasolabial cyst: Presentation of a case with a review of the literature. J Oral Maxillofacial Surg. 1984;42:18892.

2. Lassaletta AL, López RMF, Garcia AG, Balléstin C, Gallego AI, Alvarez VJ. Quiste nasoalveolar: aportación de 10 nuevos casos y revisión de la literatura. Acta Otorrinolaringologica Espanola, 1998;49:533-36.

3. Pereira Filho VA, Silva AC; Moraes M, Moreira RWF; Villalba H. Nasolabial cyst: case report. Braz. Dent. J. 2002;13(3):212-14.

4. Marcoviceanu MP, Metzger MC, Freudenberg HDN, Pautke AKC, Bettina Hohlweg-Majert. Report of rare bilateral nasolabial cysts. Journal of Cranio-Maxillofacial Surgery. 2009;37(2):83-6.
5. Yuen HW, Julian CYL, Samuel1 CLY. Nasolabial cysts: Clinical features, diagnosis, and treatment. Nasolabial cysts: Clinical features, diagnosis, and treatment. 2007;45(4):293-7.

6. Choi JH, Cho JH, Kang HJ, Chae SW, Lee SH, Hwang SJ, Lee HM. Nasolabial cyst: a retrospective analysis of 18 cases. Ear Nose hroat J. 2002;81(2):94-8.

7. Curé JK, Osguthorpe JD, Tassel PV. MR of nasolabial cysts. AJNR Am J Neuroradiol. 1996;17 (3):585-8.

8. Nixdorf DR, Peters E, Lung KE. Clinical presentation and differential diagnosis of nasolabial cyst. J Can Dent Assoc. 2003;69(3):146-9.

9. Barzilai M. Bilateral nasoalveolar cysts: case report. Clin Radiol 1994;49(2): 140-1.

10. Cohen MA, Hertzanu Y. Huge growth potential of the nasolabial cyst. Oral Surg 1985;59:441-5.

11. López-Ríos F, Lassaletta-Atienza L, DomingoCarrasco C, Martinez-Tello FJ. Nasolabial cyst: report of a case with extensive apocrine change. Oral Surg Oral Med Oral Pathol Oral Radiol Endod. 1997;84(4):404-6.

12. Neville BW, Damm DD, Allen CM, Bouquot JE. Oral and Maxillofacial Pathology. 2nd ed. Filadelfia: Saunders. 2002;25-6.

13. Su CY, Chien CY, Hwang CF. A new transnasal approach to endoscopic marsupialization of the nasolabial cyst. Laryngoscope. 1999;109(7 Pt 1): 116-8.

\section{CORRESPONDENCIA}

Rodrigo López Alvarenga

C/ Gomera, 6, esc. 2, $3^{\circ} \mathrm{H}$

04770 Adra. Almería

E-mail: mre_lopez@hotmail.com 